Individual differences and emotional inferences during reading comprehension

Christelle Gillioz and Pascal Gygax

University of Fribourg, Switzerland

Isabelle Tapiero

University of Lyon II, France 


\begin{abstract}
This paper investigated readers' representations of the main protagonist's emotional status in short narratives, as well as several mental factors that may affect these representations. General and visuo-spatial working memory, empathy and simulation were investigated as potential individual differences in generating emotional inferences. Participants were confronted with narratives conveying information about the protagonist's emotional state. We manipulated each narrative's target sentence according to its content (emotional label vs. description of the behavior associated to the emotion) and to its congruence to the story (matching vs. mismatching). The results showed that globally the difference between reading times of congruent and incongruent target sentences was bigger in the behavioral than in the emotional condition. This pattern was accentuated for high visuo-spatial working memory participants when they were asked to simulate the stories. These results support the idea that mental models may be of a perceptual nature and may more likely include behavioral elements than emotion labels per se, as suggested earlier by Gygax et al. (2007). Keywords: text comprehension, emotional inferences, self-paced reading paradigm, individual differences
\end{abstract}


Individual differences and emotional inferences during reading comprehension Research on reading comprehension has shown that readers build mental models (Johnson-Laird, 1983) or situation models (Kintsch \& van Dijk, 1978) of a scene depicted in a text that comprise both explicit and implicit elements. The latter are based on general knowledge and are referred to as inferences. Inferences generated during reading are often considered necessary to allow readers to maintain a certain local as well as global coherence of the text (Graesser, Singer, \& Trabasso, 1994). Those establishing or maintaining local coherence connect adjacent constituents of the text, whereas those establishing or maintaining global coherence connect most constituents of the text by deeper features such as the theme of a narrative. The construction of a mental model is incremental, as readers continually update their representations with new information, either explicit or implicit (Garnham \& Oakhill, 1996). If mental models can be relatively complex, they are nonetheless most likely tributary to readers' limited processing capacities (e.g., Baddeley, 1996), which may limit possible inferences as the text is being processed (i.e., on-line). Not surprisingly, research on inferences has repeatedly tried to identify which inferences are made on-line, and which are not, leading to a certain controversy on the actual need to make certain inferences.

In this paper, we focus on the mental representation readers construct of the affective state of the main character in short narratives, which has typically been subject to controversy as to whether it was inferred on-line or not. If some theories suggest that this type of inference may not be drawn during reading (e.g., the minimalist view of reading from McKoon and Rattcliff, 1992), others consider it essential for global coherence, giving it an on-line status (e.g., the constructionist view of reading by Graesser et al., 1994). Although of prime concern in early research on the matter, the relevance of these theories has been questioned with regards to the complexity of the processes involved in emotional inferences (e.g., Gygax, Tapiero, \& Carruzzo, 2007). Most importantly, and this paper furthers this idea, individual 
differences may well modulate the actual integration and complexity of the main character's emotional status in readers' mental models.

Gernsbacher, Goldsmith, and Robertson (1992) were among the first to conduct a series of experiments investigating readers' ability to mentally represent the main character's emotional status described in short narratives. In their first two experiments, presenting participants with different narratives portraying emotion-eliciting situations, they found that target sentences were read significantly faster when they contained matching emotion terms than when they contained mismatching emotion terms of opposite valence (Experiment 1) or similar valence (Experiment 2). Gernsbacher et al. (1992) concluded that readers do integrate specific emotions in their mental models while reading. These findings have been further supported by other studies investigating readers' inclusion of the main characters' affective status in their mental models (Gernsbacher, Hallada, \& Robertson, 1998; Gernsbacher, Robertson, Palladino, \& Werner, 2004; de Vega, Leon, \& Diaz, 1996).

Using short stories based on Gernsbacher et al. (1992), Gygax, Oakhill, and Garnham (2003) and Gygax, Garnham, and Oakhill (2004) questioned the assumed notion of specificity (i.e., readers infer a specific emotion label and differentiate between similar emotions or merely infer a broader feeling). They showed that although participants were sensitive to congruence manipulations of target sentences containing emotion words (i.e., they read sentences containing congruent target emotion words faster than incongruent ones), they were equally fast when reading target sentences containing (a) Gernsbacher et al.'s initial emotion words (e.g., depressed), (b) emotion words synonymous to the original ones in Gernsbacher et al. (e.g., miserable) and (c) emotional terms that were merely similar to them (e.g., useless). Most importantly, these findings were independent of the length of the narratives, reflected in the amount of information given about the main protagonist's emotional status (Gygax et al., 2004). 
Gygax et al. (2007) tried to give an explanation to account for such a seemingly nonspecific representation of the main character's emotional status. Their core argument was that most readers, under normal conditions, may only include part(s) of the emotion construct in their mental models. By emotion construct, the authors referred to Scherer's (e.g., 1987, 2005) definition of an emotion as a process of coordinated changes in the five organismic subsystems (information processing, support, executive, action, and monitoring), resulting from the appraisal of an event, internal or external. Each subsystem is associated with a particular component of emotion (i.e., cognitive, psychophysiological, action tendency, motor expression, or subjective feeling), the sum of all component changes resulting in a specific emotion. Although a more detailed account of this theory goes beyond the scope of this paper, as the construction process is dynamic and cumulative (Ellsworth \& Scherer, 2003), Gygax and colleagues $(2003,2004,2007)$ suggested that when reading about emotional situations, readers may well build a representation of emotion in an incremental manner but not inevitably reach some specific emotion (or specific emotion label such as sad or happy). The latter stage only appears if all necessary components are presented in the text or implied by it, or under critical conditions, some of which are examined in the present study. Under normal reading conditions, and if the text is rather under-specified, readers might only include the core and easy-to-represent elements of the main protagonist's emotional response. Among these elements, valence may be a good candidate, although not on its own (Gernsbacher et al., 1992), as well as behavioral responses (i.e., similar to Scherer's action tendency or motor expressions) elicited by the emotional situation. By behavioral responses the authors referred to any movement, or reference to movement in response to the emotion-eliciting situation. For example, someone might clench their fist in response to a frustrating situation or freeze in response to a scary one. Gygax et al. (2007) suggested that behavioral responses were easier to infer, at least easier than a fairly complex and abstract representation of emotion. To test 
this, they presented participants with stories in which the main character was experiencing an emotion-eliciting situation. The final target sentence of each story comprised either an emotion word, congruent or incongruent, as in previous research, or some congruent or incongruent information about the physical behavior of the main character (see Table 1 for an example).

Results indicated a significantly larger congruence effect in the behavior condition compared to the emotion condition, suggesting faster processing of congruent behavioral information as well as slower processing of incongruent one. Gygax et al. (2007) added a specific control condition for what they called the context integration problem. In short, they argued that differences between reading times of sentences containing congruent information and sentences containing incongruent information might not necessarily mirror inferential processes, as previously assumed. When reading the sentence The weather is nice, readers might infer that the sun is shining, but it is unlikely, under normal circumstances, that they infer some information about, let's say, taking the train. However, Gygax et al. (2007) argued that after the sentence The weather is nice, the sentence John is taking the train should still be read faster than It is very dark (which is incongruent), maybe in the same way that congruent emotions are read faster than incongruent ones. The former effect (i.e., John is taking the train vs. It is very dark), although similar to effects that have led researchers to believe in inferential processes, might just mirror integration processes, meaning that as long as the sentence does not contradict the context, readers maintain a fast reading pace (see Gygax et al., 2007 for a more complete argumentation). Table 2 shows an example of the control condition.

The results of the control condition were very similar (i.e., congruence effect) to the ones found in the emotion label condition, but much smaller than the difference found in the behavioral condition, hinting at the idea that emotion labels were not necessarily included in 
readers' mental representations of emotions, and that some behavioral information was likely included in readers' mental representations. Gygax et al. (2007) further suggested that such information was connected to what could be referred to as an emotion construct. In essence, readers construct a representation foundation, formed of stereotypical, or common information (i.e., behavioral reaction) associated with the emotion realm. This last point is particularly important, as Gygax (2010) showed that such a behavioral inference was not to be mistaken with any behavioral inference, independent of emotional content. They showed that although readers do generate behavioral inferences such as picking up after the sentence John saw a penny on the floor, they still showed higher congruent vs. incongruent effects in the emotion behavioral condition.

In all, these results suggest that (a) readers do infer emotional information during reading, but (b) the components included in readers' mental models are only a part of a more complex emotion construct. Given this idea of complexity, it seems reasonable to assume that there might be individual differences that may affect the construction of more elaborate emotional inferences. In the present paper, we tested this idea by specifically focusing on factors that could lead readers to form a more complex and abstract representation of emotions. Specifically, we attempted to identify three factors that may affect the elaboration of complex representations of emotions: (a) simulation, (b) empathy and (c) processing limitations (i.e., working memory). The first two, although based on different constructs (e.g., Tan, 1994), are directly associated with the possible ways people understand emotions, and the last one is related to more general cognitive functions. We do not have any strong evidence to assume interactions between these factors. For example, to our knowledge, the link between empathy and working memory has been quite difficult to establish (e.g., Hansen, 2011). We therefore only present specific predictions as to their respective effects on emotional inferences. 


\section{Simulation and Empathy}

In this paper, we embrace the idea, introduced by Oatley (1994), that through simulation (to differentiate from empathy, as discussed later), readers identify with the characters and dynamically adopt their goals and actions through their own planning process. This is a creative process by which readers map their own emotional responses onto those of the characters. It also mirrors Goldie's (1999) suggestion that people can understand emotion through in-his-shoes imagining, projecting their own beliefs and thoughts onto the character.

Simulation processes have been shown to be relevant in psycholinguistics, as the way individuals perceive real-world situations may have a large effect on word interpretation (Johnson-Laird, 1983). Situation models may hence be defined as experiential (perception and action) simulations of described situations (Barsalou, 1999; Zwaan, 2004). Recent empirical evidence converges in suggesting that comprehenders can be viewed as immersed experiencers: readers experience information as if they were participating in the activity described (Zwaan, 1999a, 1999b, 2004; Zwaan \& Rapp, 2006).

We believe that forcing an experiential way of reading may reinforce the behavioral component of the emotional inference generated during reading. More specifically, we expect the congruency difference (i.e., congruent faster than incongruent) between the emotional and the behavioral component conditions found in Gygax et al. (2007) to be more pronounced under simulation enforcement than under no specific simulation instructions. To evaluate this issue, we enforced readers in simulation processes by giving participants explicit instructions to simulate the narratives, i.e., to put themselves in the main character's shoes.

However, if we believe that enforced simulation processes could result in a more pronounced representation of behavioral information, empathic readers may construct complex representations of emotions including more specific emotional labels, regardless of simulation processes. 
Tan (1994) suggested that through empathy, which does not require the readers to share similar feelings as the protagonist (i.e., which they do when simulating), readers easily understand and imagine the main protagonist's emotional status. If, according to Goldie (1999), readers can maintain a certain observer's position when involved in empathic processes, they still anticipate how the main protagonist is going to behave and how he or she is going to feel. Some researchers on emotional inferences have strongly suggested that empathy was an important factor in the representation of emotions during reading (e.g., Komeda \& Kusumi, 2006). More empathic readers may therefore build complex representations of emotions. Again, by complex, we essentially mean that the mental models built by readers incorporate more elements of the emotion construct (i.e., not just behavioral elements) the sum of which may even activate specific emotional labels. If empathy has a role in the complexity of readers' mental representation of the main protagonist's emotional status, we expect that a measure of empathy (e.g., Interpersonal Reactivity Index; Davis, 1980, 1983) should enable us to associate different levels of empathy with differences in the complexity of emotional inferences. In essence, compared to the low empathic readers, we expect the more empathic ones to show an increased congruency effect in the emotion condition (i.e., more specifically emotion label inference) even to the extent of resembling the strong congruency effect in the behavioral condition.

Inherently, however, the very notion of disparity in the elaboration of mental models also raises issues of processing capacities, independent, or in conjunction with the empathic or simulation processes that readers may trigger.

\section{Processing Limitations: Working Memory Capacities}

Although this issue was never explicitly explored, the unspecific nature of emotional inferences suggested by Gygax and colleagues (2003, 2004, and to a certain extent 2007) could have been an artifact of readers' limited working memory capacity. This assumption 
relies on the critical idea that the mental representation of a text is partly computed and stored in working memory (Just \& Carpenter, 1992). In fact, Estevez and Calvo (2000) found that the time it took participants to generate elaborative inferences (i.e., inferences that are not essentially needed for coherence) depended on their working memory capacity. As readers with poor working memory capacity encounter new sentences or group of sentences, they may not have enough time to generate the inferences attached to the preceding sentences. Accordingly, readers do not always generate these elaborative inferences. If they do so, the inferences might be neither strong nor specific. In the same line of thinking, van den Broek, Young, Tzeng, and Linderholm (1999) suggested that readers have a limited amount of possible activation available. This means that the number and specificity of possible inferences made while reading is limited. Even if readers make the appropriate inferences, the specificity of some previously activated inferences might still diminish as readers encounter new words, sentences or propositions.

If one considers that readers, as suggested earlier, engaging in an experiential way of reading may have an accentuated representation of behavioral elements, we could hypothesize that both general working memory and visuo-spatial working memory may play a role in the elaboration of these inferences, though a different one.

On the one hand, visuo-spatial working memory limitations may hinder a representation of behavioral elements, because both visuo-spatial working memory and the conceptual representation of sensory elements share the same sensory space (Vermeulen, Corneille \& Niedenthal, 2008). This is of course based on the premise that behavioral elements of emotional inferences are sensory based. Interestingly, except for research focused on spatial text processing (Meneghetti, Gyselinck, Pazzaglia \& De Beni, 2009), the impact of visuo-spatial working memory capacities on reading comprehension has not received much support (e.g., Oakhill, Yuill \& Garnham, 2011). Still, we believe that to transpose themselves 
in the main character's shoes and experience each situation, readers might activate different visual and spatial processes relying on their visuo-spatial working memory capacities. Those with higher visuo-spatial working memory scores may therefore show an accentuated prominence of behavioral information, reflected in an even more pronounced congruency effect in the behavioral condition compare to the emotional one.

On the other hand, better general working memory scores may result in a more elaborate and complex representation of emotion (i.e., readers have more resources to process all emotion components), signaled by an increased congruency effect in the emotion condition (i.e., more specific emotion label).

\section{Method}

The present experiment was composed of three phases. The first phase comprised the main experiment (self-paced reading task), divided in two parts corresponding to the two different simulation conditions. In Part I, no specific instructions except that of reading the texts at a natural pace were given to the participants. In Part II, participants were asked to simulate, that is to imagine themselves in the main character's shoes. Each participant did both parts. The second phase was aimed at the evaluation of each participant's working memory capacities, both general and visuo-spatial working memory capacities. In the third and final phase, we evaluated each participant's level of empathy.

\section{Participants}

Eighty-six students ( 57 women and 29 men) from the University of Fribourg took part in this experiment for course credits. All participants were native French speakers. Due to unusually aberrant long reading times (i.e., several reading times above eight seconds for three- to four-words sentences), three participants were removed from the analyses. They obviously did not understand the instructions.

\section{Materials}


Experimental stories. Twenty-four stories, in French, from Gygax and Tapiero (2003) were used in this experiment. To construct these stories, Gygax and Tapiero (2003) tried to distance themselves from the habitual way stories are set up in this line of research. Most often, these stories are created by having researchers initially write (intuitive) stories that involve the main protagonist in some emotion triggering situation and then asking "judges" to agree on whether the stories rightly encompass the emotional situations (e.g., Gernsbacher et al., 1992; de Vega et al., 1996). This procedure offers researchers a certain control over linguistic factors such as frequency or syntactic properties of the sentences they create, although the content of the stories may not encompass the most complete, or salient description of emotions (or emotion situations), at least not for the population under scrutiny. Instead of creating the stories themselves, Gygax and Tapiero (2003) directly asked 30 students to each generate 24 stories focused on specific given emotions. A categorical analysis of participants' responses enabled the authors to construct 24 stereotypical stories using the most recurrent categories and the most salient wording. Although it meant that the control over certain linguistic factors was more difficult, the resulting stories correspondedsemantically as well as in the particular wording of the constituent sentences - more accurately to the population under investigation (see Table 1 for an example story).

As others have done (e.g., Gernsbacher et al., 1992; Gygax et al., 2003, 2004), in our materials, each emotion (i.e., each story) was paired with it's opposite (sad - happy). Each story was present in four different versions, depending on the target sentence. The target sentence contained a matching emotion, a mismatching emotion (from its opposite story), a matching behavioral description or a mismatching behavioral description (from its opposite story). Eight lists were constructed, to ensure that each participant would see all conditions without (Part I) and with (Part II) simulation instructions, and that each passage was present in each simulation condition across the experiment. This means that in four lists, a particular 
passage appeared in Part I and in the other four lists in Part II. In each of these four lists, it appeared with a different target sentence. A participant was presented with one of the eight lists and saw a narrative only once.

Filler stories. Twenty-four filler passages from Gygax et al. (2007) were also used. Out of the twenty-four filler stories, twelve were used to test the neutral match/mismatch question mentioned earlier. Six of these stories had a matching second sentence and six had a mismatching second sentence. In the latter case, the rest of the story was written so as to clarify the ambiguity raised by the presence of mismatching information (see Table 2 for examples of filler stories). We used the second sentence as the target sentence to ensure that the matching neutral information was unlikely to have been incorporated in readers' mental representations of the text (i.e., after just one sentence). A pilot study (in Gygax et al., 2007) ensured that the matching neutral sentences were indeed neutral and that the mismatching sentences were considered mismatching given the context.

In total, forty-eight passages were presented to each participant. In each simulation part of the experiment, there were twelve experimental stories, six experimental filler stories and six normal filler stories. For each participant, these were presented in a random order.

The stories were presented on a Power Macintosh 4400 using Psyscope Software (Cohen, MacWhinney, Flatt, \& Provost, 1993). Responses to the target sentences of each story were collected using a response button box attached to the computer, which permits millisecond accuracy.

Working memory tests. Two working memory tests were used in this study. First, participants' general working memory was evaluated with Daneman and Carpenter's (1980) French version of the Reading Span Test (RST) (Delaloye, Ludwig, Borella, Chicherio, \& de Ribaupierre, 2007). Second, participants' visuo-spatial working memory was assessed using a standardized version of Corsi Block-Tapping Task (Kessels, van Zandvoort, Postma, 
Kappelle, \& de Haan, 2000). In this task, the experimenter presents participants with a small platform composed of nine blocks. The experimenter then taps particular sequences of blocks, which participants have to reproduce. The initial sequence is composed of only two blocks. If participants reproduce correctly one of two sequences of the same length, they are presented with a sequence in which one block is added. If none is reproduced correctly, the number of correct blocks reproduced last is taken as a measure of participants' visuo-spatial working memory span. The total score is the product of the span and the number of correct trials.

Empathy scale. Each participant's level of empathy was assessed with a French version of the Interpersonal Reactivity Index (IRI) ${ }^{1}$ of Davis $(1980,1983)$. Composed of twenty-eight items, this questionnaire measures four components of empathy: Empathic Concern, Perspective Taking, Fantasy and Personal Distress. The Perspective-Taking scale and the Fantasy scale represent the cognitive facet of empathy and respectively measure the tendency to adopt the psychological point of view of others and the tendency to transpose themselves into the feelings and actions of fictitious characters. The Empathic Concern scale and the Personal Distress scale represent the emotional facets of empathy and measure otheroriented and self-oriented feelings. The total score of empathy was calculated by summing the Empathic Concern, Perspective Taking and Fantasy scales, as proposed by Pulos, Elison and Lennon (2004). The higher the score, the more empathic a participant is.

\section{Procedure}

Each story was presented one sentence at a time. As in Gygax et al. (2007), participants were instructed to read each sentence at a normal reading speed, as if they were at home, reading a magazine. They were asked to press the yes button when they finished reading a sentence, resulting in the next sentence appearing on the screen after 500 milliseconds. To make sure that participants read the story carefully, some stories $(n=16)$ were followed by a question related to the text. Participants answered the question by pressing 
either the yes or no button. After the presentation of a passage, participants were prompted with the message Are you ready? followed by the next passage. Before the actual experiment, participants underwent a practice session made up of three passages.

After completion of Part I (no specific instructions to simulate), participants were asked to rate, on an 8-point scale $(0=$ no simulation at all to $7=$ totally engaged in a simulation process), the extent to which they tried to naturally simulate the stories. After completing the scale, they were informed that similar stories would appear on the screen, but this time, they were explicitly asked to mentally simulate the situations presented to them. More specifically, we told them to make an effort to put themselves in the protagonist's shoes, as if they were living the story from the inside. At the end of Part II, participants filled the 8-points scale again, to ensure that participants complied with the instructions.

The order no instructions to simulate $\rightarrow$ instructions to simulate was fixed, as the reversed order would have made little sense, as any resulting lack of effect would be imputable to a spill-over effect. Conversely, a fixed order has the disadvantage of confounding a possible order effect by which a particular condition might be altered whereas another one might not. To ensure that this would not be the case, we examined Gygax et al.'s (2007) data by splitting them into two parts. As shown in Figure 1, both parts showed the exact same pattern in terms of the central conditions (Emotion and Behavior) and in terms of the differences in (residual) reading times between congruent and incongruent information, ruling out any confounding order effect that have had targeted the central conditions differently.

Participants' working memory capacities were then evaluated with the RST followed by the Corsi Test. Finally, participants filled in the IRI questionnaire.

\section{Results}

\section{Data Transformation}


Reading times collected in the self-paced reading task were first transformed in order to allow for individual differences in reading speed as well as to allow for the fact that all target sentences were not in the same position in the text nor of the same length. The transformation procedure was inspired by Trueswell, Tanenhaus, and Garnsey (1994) and Gygax et al. (2007). For each participant, a regression equation of time (i.e., reading time) against length (i.e., number of characters in the target sentence) was produced, separately for the 2nd (control condition) and 5th (experimental conditions) sentences. Residual reading times were calculated by subtracting the actual reading times from the reading times predicted by the regression equation. A negative residual time means that the reading time was longer than expected. Figure 2 shows the residual reading times for the different conditions (without considering individual differences). Two emotion target sentences and two behavior target sentences were removed from the analyses as they showed an awkward reverse congruence effect, hinting that their content was considered highly ambiguous by our participants.

We present the analyses in two parts. We initially present a first model that is comparable to Gygax et al. (2007). In essence, as Gygax et al. (2007) did not account for individual differences, we wanted to match our data to theirs first. We then present analyses that take individual differences into account. As this paper was exclusively concerned with individual differences, all analyses were conducted considering participants as random factor. In all analysis sections, when the main or interaction effects are not reported, it means that they were not significant.

\section{Inference Elaboration - First model}

A general 2 (Simulation: With vs. Without Simulation) X 3 (Nature: Emotion vs. Behavior vs. Control) X 2 (Congruence: Congruent vs. Incongruent) repeated measures ANOVA on the residual reading times showed a main effect of Congruence, $F(1,82)=$ $105.37, p<.001$, congruent sentences being read on average 450 milliseconds faster than 
incongruent ones, and an interaction effect of Nature by Congruence, $F(2,164)=7.52, p<$ .001. There was no other main or interaction effect. Our simulation manipulation did not produce any of the expected effects, even if the participants reported having simulated much more in Part II $(M=5.51)$ than in Part I $(M=2.96), t(80)=13.62, p<.01$.

We explored the source of the Nature by Congruence interaction effect - the main focus of Gygax et al. (2007) - in separate follow-up ANOVAs. In all follow-up analyses, Fvalues were calculated using the original model error term (Kirk, 1995). Degrees of freedom were adapted accordingly. All p-values were also converted to account for multiple comparisons (i.e., Bonferroni corrections). In the first follow-up we compared the emotion and behavior conditions in terms of congruency. For this purpose, we examined the interaction of Congruence with respect to two of the three values of the Nature variable. A 2 (Nature: Emotion vs. Behavior) X 2 (Congruence: Congruent vs. Incongruent) repeated measure ANOVA showed a significant interaction effect, $F(1,164)=6.30, p<.017$. A second follow-up ANOVA showed a significant 2 (Nature: Control vs. Behavior) X 2 (Congruence: Congruent vs. Incongruent) interaction effect, $F(1,164)=14.56, p<.017$. Finally, there was no 2 (Nature: Emotion vs. Control) X 2 (Congruence: Congruent vs. Incongruent) interaction effect, $F(1,164)=1.70, \boldsymbol{p}=. \mathbf{1 9}$. This means, as shown in Figure 2, that the difference between reading times of congruent and incongruent sentences was greater in the behavior condition (624 ms) than in the Control $(310 \mathrm{~ms})$ and the Emotion condition (416 ms). These results perfectly matched those found by Gygax et al. (2007). This is true even to the extent of the slight numerical difference between the emotion and the control condition (which will be most apparent when examining the visuo-spatial individual differences). No further post hoc analyses were performed on each condition's simple congruence effect. Essentially, they would all be significant. The crucial comparison is between the experimental conditions' congruence effects and the control condition's 
congruence effect, the latter representing a simple context yet non-inferential effect (as stressed by Gygax et al., 2007).

Further analyses were done in order to reveal any influence of participants' characteristics on emotional inference elaboration. Since we did not expect them to interact in the influence that they may have on emotional inference elaboration, each variable related to participants' characteristics was added individually to the main 2 (Simulation: With vs. Without Simulation) X 3 (Nature: Emotion vs. Behavior vs. Control) X 2 (Congruence: Congruent vs. Incongruent) analysis. Different models were tested, each by running separate ANOVAs.

\section{Inference Elaboration - Individual differences}

To investigate the role of individual differences, we explored the data by conducting hierarchical clusters on each of our measures using Ward's Method (see Table 3 for a summary of all individual differences scores). This method enabled us to classify participants in a meaningful way - more than with a manual split at least, where participants have to be shifted relatively subjectively to one of the group -, as it maximizes similarity of members of a group according to the scores of each individual on a particular scale. Ward's method is distinct from all other clustering methods because it uses an analysis of variance approach to evaluate the distances between the clusters. Basically, a score is entered into a cluster if it results in a minimal increase of the error sum of squares. In sum, Ward's method minimizes the total within-cluster variance whilst maximizing the between-cluster variance (Ward, 1963). Subsequent analyses were tested with two groups, except if stated otherwise. All cluster analyses are briefly presented, but only significant impact to the initial 2 (Simulation) X 3 (Nature) X 2 (Congruence) analysis are presented.

General working memory. The cluster analysis on the proportion of RST correct items revealed two uneven groups, reflecting a skewed distribution (supported by a -.70 
skewness value): a higher RST group $(n=63)$ with a mean of .76 $(S D=.09)$ and a lower group $(n=20)$ with a mean of $.46(S D=.09)$. The two groups were significantly different $\left(W_{s}\right.$ $=210, z=-6.77, p<.001)$. When introduced in the initial model as an independent variable, no effect other than those found without the variable was apparent. Due to its particular distribution and uneven sample sizes, we extracted the lower group participants and conducted another classification procedure. If this latter did improve sample sizes, with a higher RST group $(n=31)$ with a mean of $.84(S D=.04)$ and a lower group $(n=32)$ with a mean of .68 $(S D=.05)$, both group were quite similar (.3 SD under and .6 SD above the standard score). Therefore, and although significantly different, $t(61)=13.76, p<.001$, their inclusion to the model did not affect it $^{2}$.

Visuo-spatial working memory. The cluster analysis on total scores revealed a high Corsi group $(n=33)$ with a mean of $78.61(S D=9.50)$ and a low Corsi group $(n=50)$ with a mean of $49.14(S D=11.43)$ bearing significant difference $\left(W_{s}=1275, z=-7.74, p<.001\right)$. When introduced in the initial model as an independent variable, there was an additional 2 (Simulation) X 3 (Nature) X 2 (Congruence) X 2 (Corsi) interaction effect, $F(2,162)=4.59$, $p<.05$ (see Figure 3). Interestingly, when conducting two follow-up ANOVAs to examine individually the two Corsi groups, the low Corsi group was only affected by congruence, as shown by a significant Congruence effect, $F(1,81)=59.36, p<.025$, but no other main nor interaction effect. On the other hand, and compared to the first model analysis, the high Corsi group showed an additional Simulation by Nature by Congruence interaction effect, $F(2,162)$ $=4.27, p<.025$. When examining this interaction more closely in a follow-up ANOVA, there was a Nature by Congruence effect only significant in Part II, $F(2,162)=8.64, p<.025$, qualified by a Nature (Behavior vs. Emotion) by Congruence interaction effect, $F(1,162)=$ $13.41, p<.017$, and a Nature (Behavior vs. Control) by Congruence interaction effect, $F(1$, 
$162)=12.51, p<.017$, but no Nature (Emotion vs. Control) by Congruence interaction effect.

The Simulation manipulation seems to have affected the two groups differently. If the low Corsi group seems to have been mainly affected by congruence, independent of the conditions, the high Corsi group seems to have been influenced by the manipulation in our central conditions (Emotion and Behavior). Although no nature by congruence effect was apparent in the first part of the experiment, it changed in the second part, the congruence effect being much bigger in the Behavior condition in the second Part (901 ms) than in the first Part (568 ms), and than in the Emotion condition $(227 \mathrm{~ms})$. This is in line with the idea that situation models include perceptual components (Glenberg, 1999; Glenberg, Meyer, \& Lindem, 1987; Johnson-Laird, 1983) and that the ability to integrate elaborative and predictive inferences during reading depends upon visuo-spatial resources (Fincher-Kiefer, 2001; Fincher-Kiefer \& D’Agostino, 2004). Readers' situation models are perceptual in nature, and readers build them on the basis of their own perceptual analogous experiences. As in our experiment, when simulating, high visuo-spatial span participants may have relied on a greater capacity to embody themselves in the stories, thus leading them to a stronger representation of behavioral elements.

Still, note that in the Emotion condition, the congruence effect actually decreased from Part I to Part II (i.e., from 584 to $227 \mathrm{~ms}$ ) for the high Corsi group. In fact, the source of this effect is mainly in Part I, where the Emotion congruence effect is similar to the Behavior one. This might explain the general trend of a slight global numerical difference between the Emotion and the Control condition.

Empathy level. The cluster analysis revealed two groups: a higher Empathy group (n $=45)$ with a mean of $80.84(S D=6.30)$ and a lower group $(n=38)$ with a mean of $62.05(S D$ =7.96). Although both quite high, hinting as for the RST at a skewed distribution (supported 
by a -.51 skewness value), they were significantly different $\left(W_{s}=741, z=-7.82, p<.001\right)$. When introduced in the initial model as an independent variable and contrary to our expectations, no effect other than those found without the variable was apparent. As for the RST measure, different splitting strategies were adopted, none bearing any different pattern of results (see footnote $\# \mathbf{2}$ ).

\section{Discussion}

Gygax et al. (2007) found evidence that when reading narratives about a protagonist in an emotion eliciting situation, readers tend to build a representation of the main protagonist's emotional response which favors behavioral information (over emotions per se). In this experiment, we wanted to further their findings by examining circumstances that would intensify or reduce this process. We were particularly interested in individual differences that could account for the results found in Gygax et al. (2007). More specifically, we investigated whether the elaboration of emotional inferences was associated to particular identification processes, as defined by simulation or empathy, or to processing limitations, as defined by different types of working memory limitations.

If our results supported the idea that readers were in general more likely to include behavioral information than emotions per se in their mental representations of the text (as previously shown), we only found limited contributions from the different factors under scrutiny. In other words, the processes identified by Gygax et al. (2007) may well be stable and generalizable across a wide range of readers and reading strategies (e.g., simulation). If null effects are often delicate to interpret, we would still like to propose a discussion of the underlying processes that might explain the general lack of influence of these factors. This discussion may set the very foundations of future investigations on emotional inferences and may direct researchers interested in the matter towards most relevant issues. 
Before entering into the heart of this discussion, it is important to present the one factor that seemed to explain part of the variance of our simulation manipulation. More specifically, if our simulation manipulation did not show the expected general pattern, and this will be discussed later, our measure of visuo-spatial working memory showed that this general trend was not stable across participants with different visuo-spatial working memory capacities. Most importantly, the behavioral component of high visuo-spatial span participants' mental model seemed to have benefited from our simulation manipulation, whereas this was not the case for low visuo-spatial span participants. When asked to simulate, high visuo-spatial span participants showed increased sensitivity to target sentences containing behavioral elements. This supports the idea that simulating text increases the likelihood of including behavioral elements in readers' mental model, even if this only seems to be true for high visuo-spatial span readers. It also supports the broader notion that visuospatial capacities are crucial when generating elaborative inferences (e.g., Fincher-Kiefer, 2001; Fincher-Kiefer \& D'Agostino, 2004) and that, at least for some participants, when compelled to be actors in the text (i.e., in our study through a simulation manipulation), perceptual and motor information may show access and encoding facilitation (Ditman, Brunyé, Mahoney, \& Taylor, 2010). Note that the present data cannot truly disentangle the detailed processes that influence visuo-spatial working memory, but at least they suggest that visuo-spatial working memory is a serious candidate when examining processes at stake in emotional inferences and reading comprehension.

Even though low- and high- visuo-spatial participants showed differential simulation effects, the main Nature by Congruence effect remained, suggesting that low visuo-spatial span participants still favored behavioral information to emotions per se (i.e., regardless of simulation). This strongly suggests that readers transpose themselves in the protagonist's shoes during reading, even when no specific instructions to do so are given to them, leading to 
a stronger behavioral representation of the situation. In a sense, this is not surprising, considering that to understand a text and the main protagonist's mental states, readers automatically lay upon their own psycho-physiological resources to imagine themselves living the same story and performing the same actions as the protagonist (e.g., Glenberg \& Kaschak, 2002; Goldman, 2002; Mouilso, Glenberg, \& Havas, 2007; Zwaan, 2004; Zwaan \& Taylor, 2006). By simulating, readers substitute their own feelings for those of the protagonist (Goldman, 2005) and can access knowledge of what is described in the text and elaborate a mental representation based on this knowledge. Niedenthal, Winkielman, Mondillon, and Vermeulen (2009) and Havas, Glenberg, and Rinck (2007) further argued that the process of simulation is necessary to access emotional meaning and elaborate mental representations of emotional texts.

In some respects, one could argue that the general lack of effect of our simulation manipulation reflects a ceiling effect, all participants being more or less already engaged in simulation processes. This is compared with research suggesting that even passive reading of action-related words already activates motor- and premotor-associated brain regions (e.g., Hauk, Johnsrude \& Pulvermüller, 2004), implying rather spontaneous simulation processes.

A ceiling effect might also exist for both our empathy and our general working memory measures. Low- and high-empathy participants, as well as low- and high- general working memory participants showed all relatively high scores, at least compared to standards scores. In addition to this, empathy might be connected to simulation processes, some authors arguing that empathy and simulation are difficult to disentangle (Decety \& Grèzes, 2006; Goldman, 2005; Preston \& de Waal, 2002). If our results suggest that working memory and empathy may not be accountable for the relative lack of elaboration, it should be stressed that the groups of participants were quite similar. 
These probable ceiling effects raise two crucial issues. First, they raise the pertinence of a constant reliance on university students as participants. Some measures may not be most appropriate with regard to undergraduate students' characteristics. Second, and on a more conceptual level, they do support the fact that complex elaboration of emotions might not be present when inferring emotions, even for participants with relative high levels of empathy, simulation processes and working memory. According to Gygax et al. (2007), the apparent dominance of behavioral information in readers' mental models can be explained by different factors, all based on the perspective that behavioral elements are more easily activated and included in readers' mental models. First, they argued that readers do not need to construct elaborate and complex representations to attain a reasonable understanding of the text. This is in line with the idea found in research on text comprehension that underspecified representations of text are often perfectly acceptable (e.g., Sanford \& Graesser, 2006), and that good enough representations (Ferreira, Bailey, \& Ferraro, 2002) may in the long term be beneficial in terms of cognitive load. In essence, it might even be counterproductive for readers to automatically integrate elaborate and complex representations of the main protagonist's emotional status, as any potential shifts may involve a large amount of cognitive processing. Second, if one considers the main function of emotions to prepare the body for the appropriate actions (Frijda, 1986; Scherer, 1982), readers, through simulation, will most likely activate neural areas that are implicated in actions associated with the described emotions (Havas et al., 2007). Such an activation, in turn, will lead readers to the construction of mental representations mostly composed of behavioral characteristics.

Although these two explanations coincide with our results, we still believe that some situations may elicit more elaborate representations of emotions. If behavioral elements are well-suited candidates for the representation of the protagonist's emotional status, as explained above, there may still be textual elements that elicit elaborate emotions. These 
textual elements have in fact been difficult to explore, as the narratives used in the present study, as well as in studies of others, although more or less ecological, were never structured in a consistent manner regarding the quality and quantity of emotional information they conveyed. Typically, Scherer and his colleagues (e.g., 1984, 2005) identified different emotion sub-components which combination is necessary to produce specific emotional responses. In this paper, as well as in most research on emotional inferences, we may not have paid enough attention to the emotion components that the passages contained. The present results may be, in terms of the lack of specific emotional inferences, the direct result of such an issue, which may also have impacted upon the relatively moderate effects of our individual differences. We are currently investigating this very issue in our laboratory.

On a different yet related methodological note, the present experiment, as others before, was based on the habitual match vs. mismatch or congruent vs. incongruent paradigm. Fundamentally, this could be the cause of some of the null effects found in this experiment for two main reasons. First of all, in case of a match vs. mismatch effect, it is always difficult to know if the effect is mainly associated with the incongruent information (i.e., as you often find in EEG studies on the N400, for example) or with the congruent one. Second, in selfpaced reading experiments, match vs. mismatch effects are often quite important in terms of milliseconds (e.g., 200 milliseconds minimum), suggesting that the paradigm may not be sensitive to small changes of processes, as they may have appeared in this experiment.

Finally, we can not exclude the fact that the null effects reported here result from a lack of statistical power. Our results would benefit from a replication with a greater sample, as the differences between the conditions examined in this paper may have been too subtle to be demonstrated given the relative small number of participants (still higher than most of the studies on the topic) and the different variables under observation. 
In conclusion, the experiment presented in this paper confirmed previous findings (e.g., Gygax, 2010) on the unspecific nature of emotional inferences and on the prevalence of behavioral elements in readers' mental models of emotions. It also showed that visuo-spatial memory capacities might influence such predominance, but that general working memory, empathy capacities and reading preference were not of prime importance in those inferential processes. 


\section{References}

Baddeley, A. (1996). Exploring the central executive. Quarterly Journal of Experimental Psychology, 49A, 5-18.

Barsalou, L. W. (1999). Perceptual symbol systems. Behavioral \& Brain Sciences, 22, 577 660.

Cohen, J., MacWhinney, B., Flatt, M., \& Provost, J. (1993). PsyScope: An interactive graphical system for designing and controlling experiments in the psychology laboratory using Macintosh computers. Behavior Research Methods Instruments and Computers, 25, 257-271.

Daneman, M., \& Carpenter, P. A. (1980). Individual differences in working memory and reading. Journal of verbal learning and verbal behavior, 19, 450-66.

Davis, M. H. (1980). A multidimensional approach to individual differences in empathy. JSAS Catalog of Selected Documents in Psychology, 10, 85.

Davis, M. H. (1983). Measuring individual differences in empathy: Evidence for a multidimensional approach. Journal of Personality and Social Psychology, 44, 113126.

Decety, J., \& Grèzes, J. (2006). The power of simulation: Imagining one's own and other's behavior. Brain Research, 1079, 4-14.

Delaloye, C., Ludwig, C., Borella, E., Chicherio, C., \& de Ribaupierre, A. (2008). The Reading Span as a measure of working memory capacity: Norms based on a French speaking population of 775 younger and older adults. Revue européenne de psychologie appliquée, 58, 89-103.

de Vega, M., Leon, I., \& Diaz, J. M. (1996). The representation of changing emotions in reading comprehension. Cognition and Emotion, 10, 303-322. 
Ditman, T., Brunyé, T. T., Mahoney, C. R., \& Taylor, H. A. (2010). Simulating an enactment effect: Pronouns guide action simulation during narrative comprehension. Cognition, $115,172-178$.

Ellsworth, P. C., \& Scherer, K. R. (2003). Appraisal processes in emotion. In R. J. Davidson, K. R. Scherer, \& H. H. Goldsmith (Eds.), Handbook of affective sciences (pp. 572595). New York: Oxford University Press.

Estevez, A., \& Calvo, M. G. (2000). Working memory capacity and time course of predictive inferences. Memory, 8, 51-61.

Ferreira, F., Bailey, K. G. D., \& Ferraro, V. (2002). Good-enough representations in language comprehension. Current Directions in Psychological Science, 11, 11-15.

Fincher-Kiefer, R. (2001). Perceptual components of situation models. Memory \& Cognition, $29,336-343$.

Fincher-Kiefer, R., \& D'Agostino, P. R. (2004). The role of visuo-spatial resources in generating predictive and bridging inferences. Discourse Processes, 37, 205-224.

Frijda, N. H. (1986). The emotions. Cambridge: Cambridge University Press.

Garnham, A., \& Oakhill, J. V. (1996). The mental models theory of language comprehension. In B. K. Britton \& A. C. Graesser (Eds.), Models of understanding text (pp. 313-339). Mahwah, NJ: Erlbaum.

Gernsbacher, M. A., Hallada, B. M., \& Robertson, R. R. W. (1998). How automatically do readers infer fictional characters' emotional states? Scientific Studies of Reading, 2, 271-300.

Gernsbacher, M. A., Goldsmith, H. H., \& Robertson, R. R. W. (1992). Do readers mentally represent characters' emotional states? Cognition and Emotion, 6, 89-111.

Gernsbacher, M. A., Robertson, R. R. W., Palladino, P., \& Werner, N. K. (2004). Managing mental representations during narrative comprehension. Discourse Processes, 37, 145- 
164.

Glenberg, A. (1999). Why mental models must be embodied. In G. Rickheit \& C. Habel (Eds.), Mental models in discourse processing and reasoning. Amsterdam: Elsevier.

Glenberg, A. M., \& Kaschak, M. P. (2002). Grounding language in action. Psychonomic Bulletin \& Review, 9, 558-565.

Glenberg, A. M., Meyer, M., \& Lindem, K. (1987). Mental models contribute to foregrounding during text comprehension. Journal of memory and language, 26, 6983.

Goldie, P. (1999). How we think of others' emotions. Mind \& Language, 14, 394-423.

Goldman, A. I. (2005). Imitation, mind reading, and simulation. In S. Hurley \& N. Chater (Eds.), Perspective on Imitation, from Neuroscience to Social Science (Vol. 2, pp. 7993). Cambridge: MIT Press.

Graesser, A. C., Singer, M., \& Trabasso, T. (1994). Constructing inferences during narrative text comprehension. Psychological Review, 101, 371-371.

Gygax, P. M. (2010). L’inférence émotionnelle durant la lecture et sa composante comportementale. [Emotion inference during reading and its behavioral component]. L’Année psychologique, 110, 253-273.

Gygax, P. M., Garnham, A., \& Oakhill, J. (2004). Inferring characters' emotional states: Can readers infer specific emotions? Language and Cognitive Processes, 19, 613-638.

Gygax, P. M., Oakhill, J., \& Garnham, A. (2003). The representation of characters' emotional responses: Do readers infer specific emotions? Cognition and Emotion, 17, 413-428.

Gygax, P., \& Tapiero, I. (2003). Divide and conquer: A study of the semantic structure of emotional inferences in reading comprehension. Paper presented at the Annual Conference of Swiss Psychology, Bern, Switzerland.

Gygax, P., Tapiero, I., \& Carruzzo, E. (2007). Emotion inferences during reading 
comprehension: What evidence can the self-pace reading paradigm provide? Discourse Processes, 44, 33-50.

Hansen, S. (2011). Inhibitory control and empathy-related personality traits: Sex-linked associations. Brain and Cognition, 76, 364-368.

Hauk, O., Johnsrude, I. \& Pulvermuller, F. (2004). Somatotopic representation of action words in human motor and premotor cortex, Neuron, 41, 301-307.

Havas, D. A., Glenberg, A. M., \& Rinck, M. (2007). Emotion simulation during language comprehension. Psychonomic Bulletin and Review, 14, 436-441.

Johnson-Laird, P. N. (1983). Mental models. Cambridge, MA: Harvard University Press.

Just, M. A., \& Carpenter, P. A. (1992). A capacity theory of comprehension: Individual differences in working memory. Psychological review, 99, 122-149.

Kessels, R. P. C., van Zandvoort, M. J. E., Postma, A., Kappelle, L. J., \& de Haan, E. H. F. (2000). The Corsi block-tapping task: Standardization and normative data. Applied Neuropsychology, 7, 252-258.

Kintsch, W., \& van Dijk, T. A. (1978). Toward a model of comprehension and production. Psychological Review, 85, 363-394.

Kirk, R. E. (1995). Experimental design: Procedures for the behavioral sciences (3rd ed.). Pacific Grove, CA: Brooks/Cole.

Komeda, H., \& Kusumi, T. (2006). The effect of a protagonist's emotional shift on situation model construction. Memory \& Cognition, 34, 1548-1556.

McKoon, G., \& Ratcliff, R. (1992). Inference during reading. Psychological Review, 99, 440466.

Meneghetti, C., Gyselinck, V., Pazzaglia, F., \& De Beni, R. (2009). Individual Differences in Spatial Text Processing : high spatial ability can compensate for spatial working memory interference. Learning and Individual differences, 19, 577-589. 
Mouilso, E., Glenberg, A. M., \& Havas, D. (2007). Differences in action tendencies distinguish anger and sadness after comprehension of emotional sentences. In D. S. McNamara \& J. G. Trafton (Eds.), Proceedings of the 29th Annual Conference of the Cognitive Science Society (pp. 1325-1330). Austin, TX: Cognitive Science Society.

Niedenthal, P. M., Winkielman, P., Mondillon, L., \& Vermeulen, N. (2009). Embodiment of emotion concepts. Journal of Personality and Social Psychology, 96, 1120-1136.

Oakhill, J., Yuill, N., \& Garnham, A. (2011).The differential relations between verbal, numerical and spatial working memory abilities and children's reading comprehension. International Electronic Journal of Elementary Education, 4, 83-106.

Oatley, K. (1994). A taxonomy of the emotions of literary response and a theory of identification in fictional narrative. Poetics, 23, 53-74.

Preston, S. D., \& de Waal, F. B. M. (2002). Empathy: Its ultimate and proximate bases. Behavioral and Brain Sciences, 25, 1-72.

Pulos, S., Elison, J., \& Lennon, R. (2004). The hierarchical structure of the Interpersonal Reactivity Index. Social Behavior and Personality, 32, 355-359.

Sanford, A. J., \& Graesser, A. C. (2006). Shallow processing and underspecification. Discourse Processes, 42, 99-108.

Scherer, K. R. (1982). Emotion as a process: Function, origin and regulation. Social Science Information, 21, 555-570.

Scherer, K. R. (1984). On the nature and function of emotion: A component process approach. In K. R. Scherer \& P. Ekman (Eds.), Approaches to Emotion (pp. 293-317). Hillsdale, NJ: Erlbaum.

Scherer, K. R. (1987). Toward a dynamic theory of emotion: The component process model of affective states. Geneva Studies in Emotion and Communication, 1, 1-98.

Scherer, K. R. (2005). What are emotions? And how can they be measured? Social Science 
Information, 44, 695-729.

Trueswell, J. C., Tanenhaus, M. K., \& Garnsey, S. M. (1994). Semantic influence on syntactic processing: Use of thematic information in syntactic disambiguation. Journal of Memory and Language, 33, 265-312.

van den Broek, P., Young, M., Tzeng, Y., \& Linderholm, T. (1999). The landscape model of reading: Inferences and the one-line construction of memory representation. In H. van Oostendorp \& S. R. Goldman (Eds.), The construction of mental representation during reading. (pp. 71-98). Mawhah, NJ: Erlbaum.

Vermeulen, N., Corneille, O., \& Niedenthal, P.M. (2009). Sensory load incurs conceptual processing costs. Cognition, 109, 287-294.

Ward, J. H. Jr. (1963). Hierarchical grouping to optimize an objective function. Journal of the American Statistical Association, 48, 236-244.

Zwaan, R. A. (1999a). Embodied cognition, perceptual symbols, and situation models. Discourse Processes, 28, 81-88.

Zwaan, R. A. (1999b). Situation models: The mental leap into imagined worlds. Current Directions in Psychological Science, 8, 15-18.

Zwaan, R. A. (2004). The immersed experiencer: Toward an embodied theory of language comprehension. In B. H. Ross (Ed.), The psychology of learning and motivation: Advances in research and theory (Vol. 44, pp. 35-62). New York, NY: Elsevier.

Zwaan, R. A., \& Rapp, D. N. (2006). Discourse comprehension. In M. J. Traxler \& M. A. Gernsbacher (Eds.), Handbook of psycholinguistics (pp. 725-764). San Diego, CA: Elsevier.

Zwaan, R. A., \& Taylor, L. J. (2006). Seeing, acting, understanding: Motor resonance in language comprehension. Journal of Experimental Psychology:General, 135, 1-11. 


\section{Footnotes}

${ }^{1}$ Free translation, not validated, available from Stephanie Braun (sbraun@ulb.ac.be), ULB - Erasme Hospital, Brussels, Belgium.

${ }^{2}$ To ensure that the null results were not due to our categorization procedure, we also performed extra analyses adopting different strategies: (a) we used three clusters and (b) we split the data into quartiles and compared high and low quartiles. None of these bore any consequence on the analyses presented in this paper. These different strategies were also adopted with other variables when relevant. For the Empathy scale, we also tested each of its four sub-components separately, but nothing came of these different analyses. We also ensured that the null results were not due to a questionable reliability of the IRI sub-scales. This was not the case, all Cronbach's $\alpha$ being higher than .7. 
Table 1

Example of an experimental narrative used in Gygax et al. (2007) and in this experiment

Narrative:

Suzanne came back from her regular visit to the nursing home. She walked slowly from the nursing home to her place. She thought of the days with her grandmother with a heavy heart. She had trouble holding back her tears when thinking of her grandmother alone in her room.

Target sentences:

Matching emotion: $\quad$ As you could expect, Suzanne was feeling sad.

Mismatching emotion: As you could expect, Suzanne was feeling happy.

Matching behavior: She sat on her settee, wrapped in a blanket.

Mismatching behavior: She danced all night, as she was always the one to show others how to party. 
Table 2

Examples of control narratives used in Gygax et al. (2007) and in this experiment. In the first narrative, the second sentence is the congruent neutral target sentence and in the second narrative, the second sentence is the incongruent neutral target sentence.

Narrative 1: Neutral matching

Georges was writing and preparing for a conference in the East. He wanted to make the most out of his trip. He therefore planned several visits to his friends and to the people he knew on the way. He was taking his time to prepare for the trip and had been preparing since spring.

Narrative 2: Neutral mismatching

Cindy had just finished work and was going to her gym. She thought that going to the gym was individualistic and preferred volleyball. But after a knee injury, she could not play volleyball anymore. After changing, she entered the gym, which was empty and dark. "That's strange", she thought as she was warming up. 
Table 3

Mean scores (and standard deviations in brackets) of each individual differences score, after splitting our sample using the Ward method. In our sample, RST scores ranged from 25 to .94 (the total score is the product of the span and the number of correct trials), Corsi scores from 24 to 104 and IRI scores from 33 to 95.

\begin{tabular}{lccc}
\hline & High Goup & Low Group & Mean \\
\hline $\begin{array}{l}\text { General working memory } \\
\text { (RST) }\end{array}$ & $.76(.1)$ & $.46(.1)$ & $.61(.09)$ \\
\hline $\begin{array}{l}\text { Visuo-spatial memory (Corsi } \\
\text { Block-Tapping Task) }\end{array}$ & $78.61(9.5)$ & $49.14(11.4)$ & $63.88(10.5)$ \\
\hline & $80.84(6.3)$ & $62.05(8.0)$ & $71.45(7.1)$ \\
\hline
\end{tabular}


Figure 1. Mean differences in reading times between congruent and incongruent Emotion and Behavior information in Gygax et al. (2007). The slight decrease in reading time differences between Part I and Part II is independent of the conditions under investigation and reflects a certain habituation to the task by the participants.

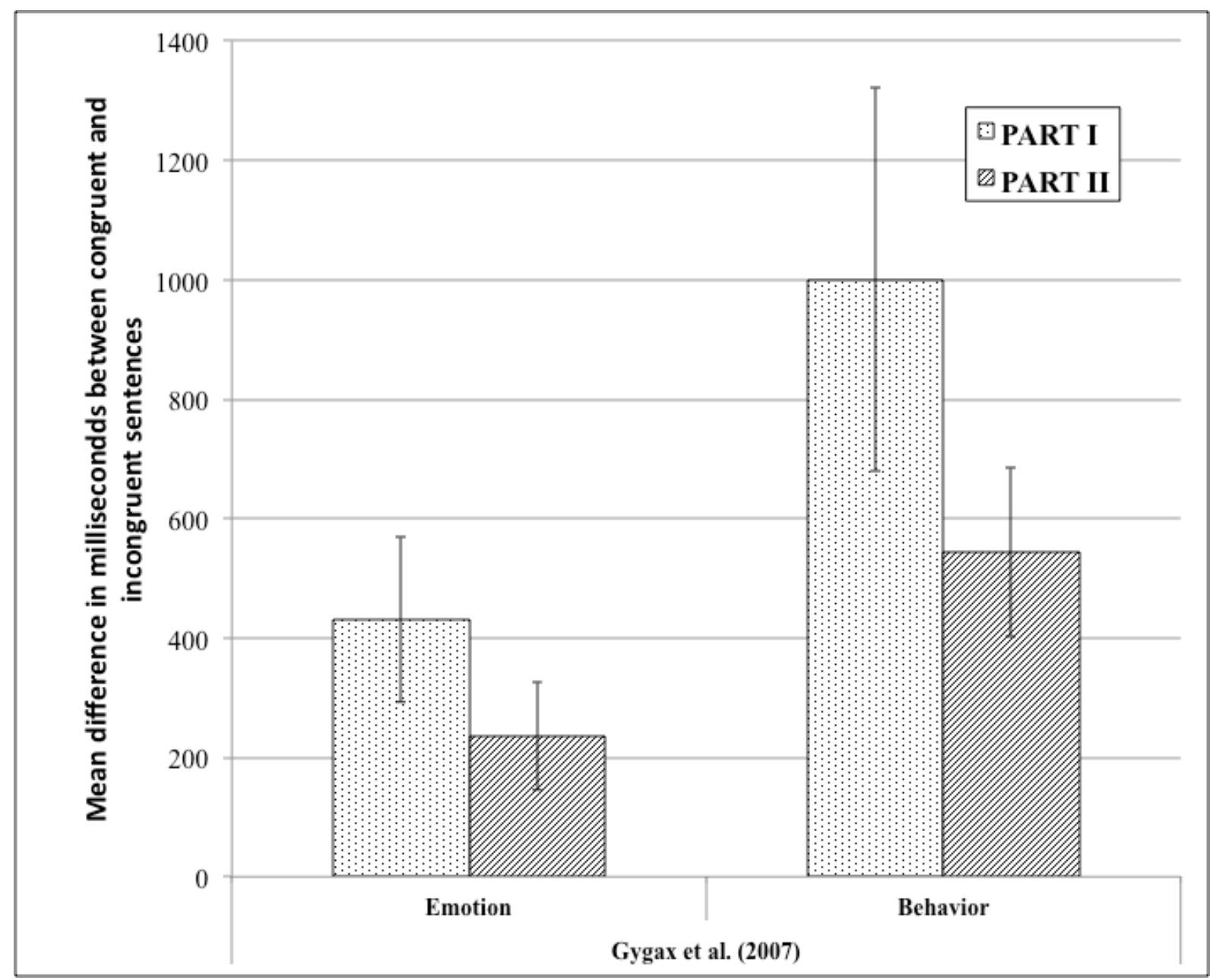


Figure 2. Mean residual times in each emotion content and simulation condition, without considering individual differences. Negative residuals mean slower reading times (as predicted by each participant's regression of time by number of letters).

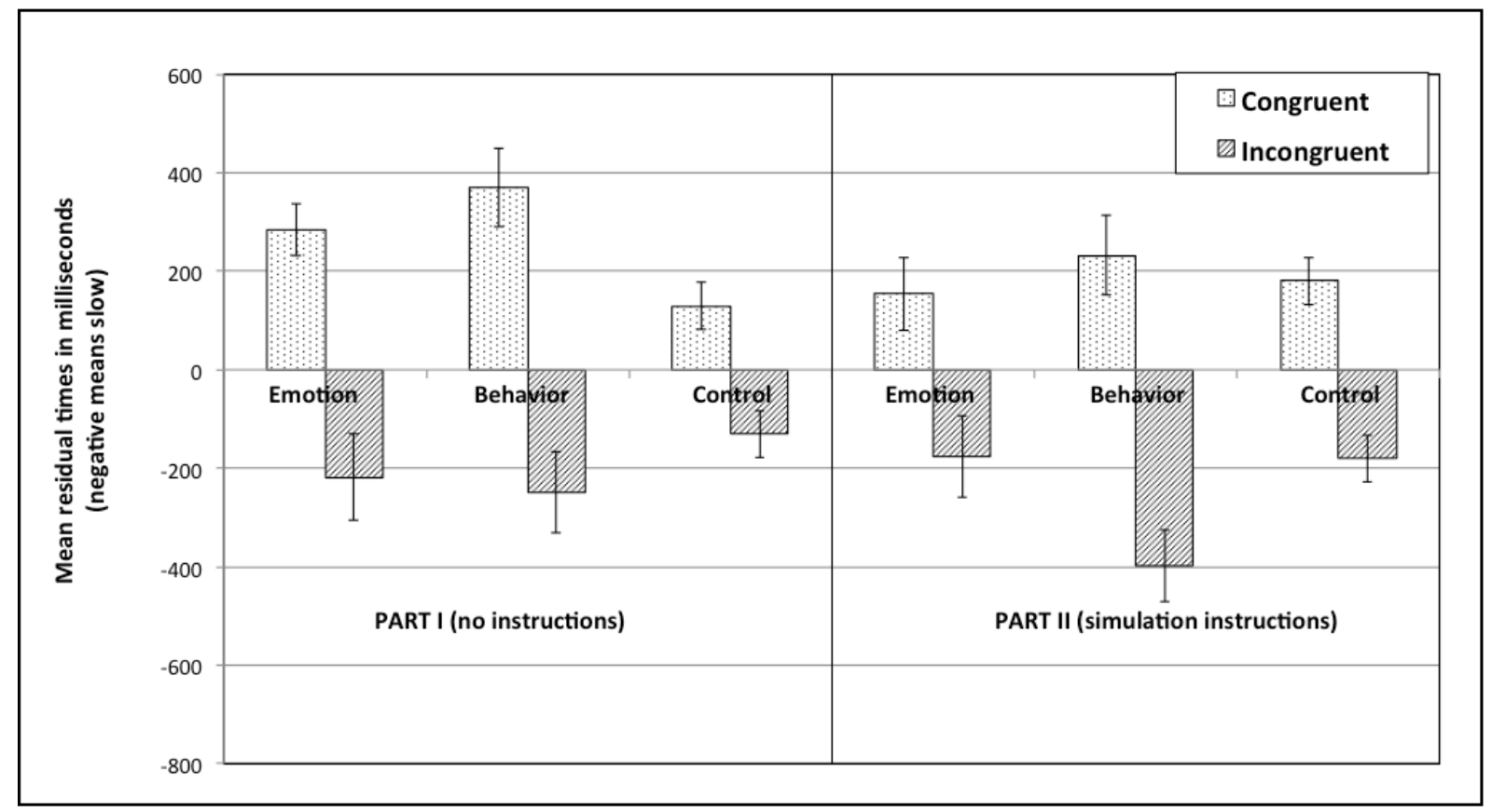


Figure 3. Mean residual times in each emotion content and simulation condition as a function of Corsi scores. Negative residuals mean slower reading times (as predicted by each participant's regression of time by number of letters).

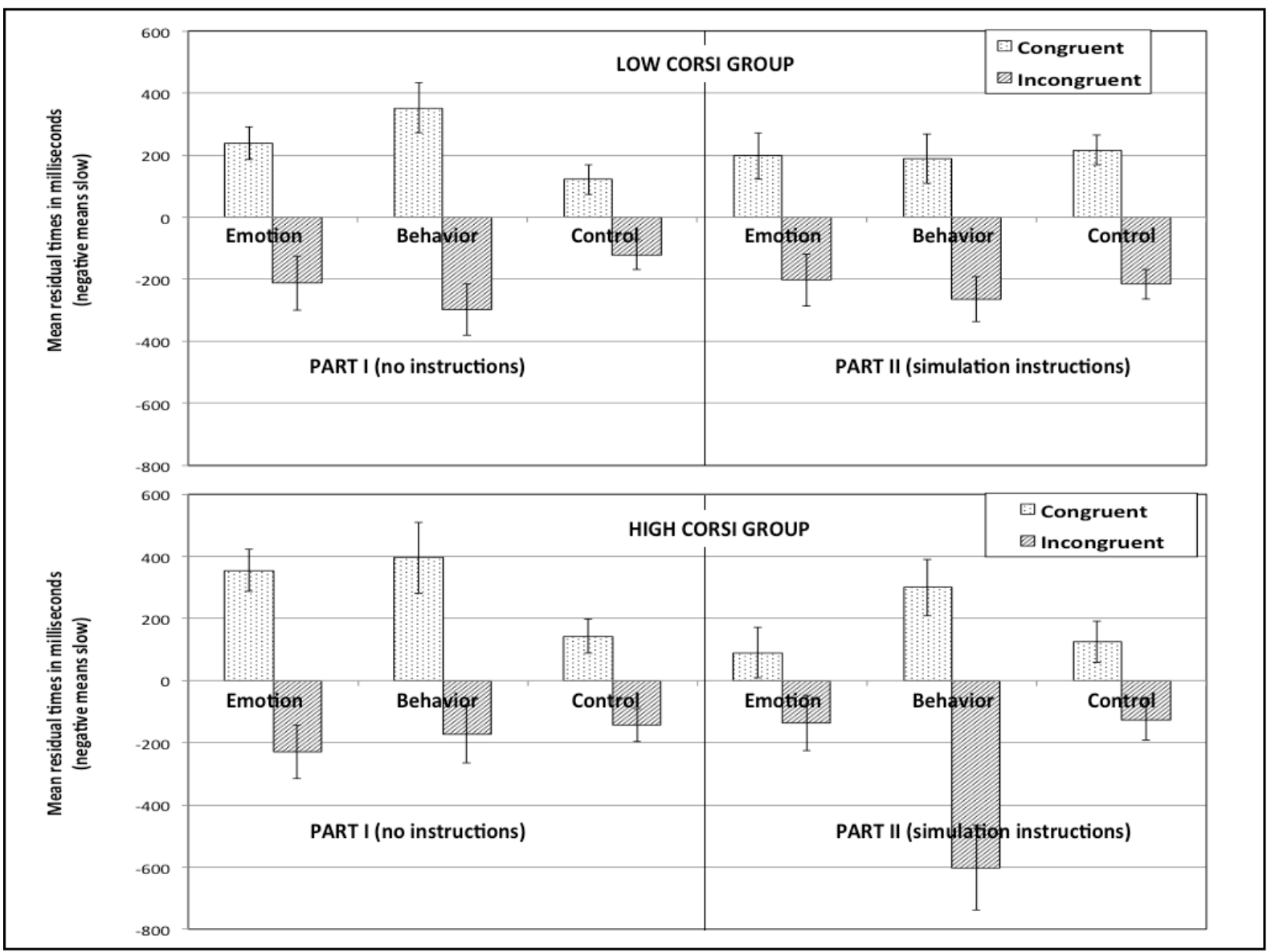

